\title{
Condições de saúde bucal de idosos institucionalizados
}

\author{
Kléryson Martins Soares Francisco*, Nayana Teixeira Silveira*, \\ Cezar Augusto Casotti'”, Douglas Leonardo Gomes Filho"***, Juciara França dos Santos ${ }^{* * * * *}$
}

\section{Resumo}

Diante do acelerado crescimento da população idosa e do aumento da expectativa de vida, é notória a necessidade de se proporcionar às pessoas um envelhecimento com qualidade de vida. Este trabalho propõe-se a verificar as condições de saúde bucal de 46 idosos institucionalizados, residentes na Fundação Leur Brito, no município de Jequié - Bahia. Os resultados mostraram uma perda dentária de $97,28 \%$, com CPO-D médio de 29,02. A necessidade do uso de prótese total superior (76,09\%) e inferior $(69,56 \%)$ demonstrou a precária condição de saúde bucal. A condição periodontal mostrou-se com prevalência de sangramento no $\mathrm{CPI}$, e perda de 0 a $3 \mathrm{~mm}$ no PIP. Quanto ao grau de dependência, observou-se que $41,30 \%$ dos idosos eram independentes. No acometimento de enfermidades, prevaleceram a hipertensão e o diabetes. As condições encontradas no levantamento revelam a precária situação da saúde bucal desses idosos, ressaltando a importância de ações voltadas para a educação em saúde e de um atendimen- to satisfatório que Ihes forneça um enveIhecer mais sereno. É necessário focar em programas assistenciais específicos para que em breve essa condição se modifique positivamente.

Palavras-chave: Envelhecimento. Saúde do idoso institucionalizado. Promoção da saúde.

\section{Introdução}

O Brasil, assim como diversos outros países, encontra-se em um processo acelerado de envelhecimento. A população idosa, composta pelos indivíduos com idade igual ou maior que 60 anos, segundo o Estatuto do Idoso (BRASIL, 2003), compõe o segmento populacional que mais cresce. Estudos apontam que, até o ano de 2025, o país possuirá a sexta maior população idosa do mundo, com mais de 30 milhões de pessoas nessa faixa etária, representando

* Mestre em Odontologia Preventiva e Social pela Universidade Estadual Paulista (Unesp). Professor assistente da Universidade Estadual do Sudoeste da Bahia (UESB). Endereço para correspondência: Av. José Moreira Sobrinho, s/n, Jequiezinho, CEP: 45206-190, Jequié - BA. E-mail: kmartins@uesb.edu.br.

** Acadêmica do curso de Odontologia da Universidade Estadual do Sudoeste da Bahia (UESB).

*** Doutor em Odontologia Preventiva e Social pela Universidade Estadual Paulista (Unesp). Professor adjunto da Universidade Estadual do Sudoeste da Bahia (UESB).

***** Mestre em Odontologia pela Universidade Federal Fluminense (UFF). Professor assistente da Universidade Estadual do Sudoeste da Bahia (UESB).

******Acadêmica do Curso de Odontologia da Universidade Estadual do Sudoeste da Bahia (UESB).

$\longrightarrow$ doi:10.5335/rbceh.2012.044 
quase $15 \%$ da população total. (SILVA et al., 2008; RODRIGUES et al., 2012).

Nas últimas décadas, tem sido constatada uma queda nas taxas de natalidade e um aumento na expectativa de vida, com consequente crescimento da população idosa. O benefício deve-se ao desenvolvimento da ciência e de novas tecnologias, muitas delas aplicadas à medicina e à odontologia, fornecendo boas condições ao tratamento das enfermidades. Esse fato torna ainda mais relevante a atenção direcionada à saúde bucal ao grupo da terceira idade. (SOUZA et al., 2001).

Em vista disto, a demanda por serviços de instituições para o atendimento e o acompanhamento integral ao idoso tem aumentado. A instalação do idoso em uma instituição de longa permanência pode apresentar-se como única solução para a família que se percebe sem suporte financeiro e psicológico. Nessas instituições, o indivíduo vive na forma de internato, por tempo determinado ou não, mediante pagamento ou não, sendo assistido por cuidadores de diversas áreas da saúde. (REIS et al., 2005).

A saúde bucal, componente essencial para a saúde geral do indivíduo, tem sido relegada ao esquecimento, quando se discutem as condições de saúde da população idosa. O edentulismo é visto pela sociedade, e até mesmo pelos cirurgiões-dentistas, como algo normal e natural com o avanço da idade, o que, no entanto, não deve ser aceito como uma verdade. (ROSA et al., 1992).

O Projeto SB-2003 Brasil, coordenado pelo Ministério da Saúde, em 2003, foi a primeira pesquisa em nível nacional a avaliar as condições de saúde bucal de idosos.
A amostra incluiu indivíduos de 65 a 74 anos de 250 municípios. Em 2010, uma nova pesquisa foi realizada, o SB Brasil 2010. Após a divulgação do relatório final desta pesquisa, será possível comparar os dados e avaliar a evolução da saúde bucal dos idosos no país. (BRASIL, 2004; BRASIL, 2010).

Contudo, é difícil estimar a futura situação da saúde bucal e as necessidades de tratamento da próxima geração de idosos com base nos dados epidemiológicos da população idosa de hoje, pois as mudanças são constantes, principalmente devido ao consumo de água fluoretada do abastecimento público e ao uso de dentifrícios. Porém, é necessário conhecer o estado de saúde bucal desse grupo etário, como também obter dados epidemiológicos que sirvam de subsídios para o desenvolvimento de programas direcionados a essa população. (COLUSSI et al., 2002; RIBEIRO et al., 2011).

O presente estudo tem como objetivo verificar as condições de saúde bucal de idosos institucionalizados do município de Jequié, residentes na instituição asilar Fundação Leur Brito (FLB).

\section{Materiais e métodos}

Este é um estudo epidemiológico transversal, realizado no período de abril a maio de 2011, o qual verificou a prevalência das lesões bucais em todos os indivíduos acima dos 60 anos residentes na FLB, no município de Jequié - BA. O campo de estudo limitou-se a essa instituição, a qual, vinculada à igreja católica, foi fundada em 1957 e abriga 57 idosos.

A coleta de dados foi realizada por meio de consultas aos prontuários dos 
idosos e de um exame clínico, mediante formulário específico contendo informações sobre características sociodemográficas (idade, sexo), hábitos de vida e exame físico intraoral.

O exame foi realizado em ambiente sob a luz natural por uma examinadora e um anotador previamente calibrados (Coefiente Kappa =0,93). Foram utilizados espelho bucal plano $\mathrm{n}^{\circ} 5$, sonda periodontal tipo ball preconizada pela Organização Mundial de Saúde (OMS), espátulas de madeira, gaze, papel toalha e uma ficha clínica. Durante a realização do exame, a examinadora estava devidamente paramentada com gorro, óculos de proteção, luva, máscara e jaleco.

Para avaliar a prevalência da cárie, foi utilizado o Índice de CPO-D, que indica o número médio de dentes cariados, perdidos e obturados. O uso e a necessidade de prótese foram analisados de acordo com a presença ou não dessa peça (em bom estado) no momento do exame.

Para analisar a condição periodontal, foi utilizado o Índice Periodontal Comunitário (CPI), seguindo os códigos: $\mathrm{X}$ - sextante excluído, código 0 - sextante hígido, código 1- sextante com presença de sangramento em pelo menos um dos dentes, código 2- presença de cálculo supra- ou subgengival, código 3- bolsa periodontal de 4 a $5 \mathrm{~mm}$ e código 4 - bolsa periodontal com $6 \mathrm{~mm}$ ou mais profunda.

Ainda na análise da condição periodontal, foi utilizado o Índice de Perda de Inserção Periodontal (PIP), que avalia a retração do tecido gengival quanto à junção amelocementária (JAC). Para uma distância entre 0 e $0,5 \mathrm{~mm}$ do tecido gengival em relação à JAC, considerou-se ausência de retração gengival (código
0). Para os códigos de I a IV, a presença de retração gengival foi considerada da seguinte forma: I- entre $0,5-3 \mathrm{~mm}, \mathrm{II}-3-5$ $\mathrm{mm}$, III-5-7 $\mathrm{mm}$ e IV- maior que $7 \mathrm{~mm}$.

Além do exame clínico bucal, foram avaliadas as condições psicomotoras dos idosos, por meio do grau de dependência (dependente, parcialmente dependente e independente). Posteriormente, foram levantados os problemas de saúde por meio de consulta aos prontuários do departamento médico da FLB. Juntamente a esses dados, foi possível ter acesso à data de entrada do idoso à FLB.

Os dados obtidos foram tabulados e analisados nos softwares Epibuco ${ }^{\circledR}$ (Eymar Souza Lopes), Statical Package for Social Sciences ${ }^{\circledR}$ (SPSS) na versão 21 e Office Excel ${ }^{\circledR} 2007$.

Este estudo foi aprovado pelo Comitê de Ética de Pesquisa da Universidade Estadual do Sudoeste da Bahia (Protocolo $\mathrm{n}^{\mathrm{o}}$ 192/2010).

\section{Resultados}

Foram examinados 46 idosos residentes na Fundação Leur Brito, os quais apresentaram uma média de 79,5 anos, sendo $52,18 \%$ (24) do gênero feminino. A faixa etária ficou assim dividida: 12 indivíduos com idade entre 60 e 74 anos e 34 acima de 75 anos.

A Tabela 1 revela os dados do CPO-D e o valor percentual de seus componentes, de acordo com o gênero e a faixa etária. A análise dos componentes do CPO-D evidenciou a grande quantidade de dentes extraídos, tendo todos os participantes apresentado perda dentária de mais de uma unidade. 
Tabela 1 - Índice CPO-D e percentagem de dentes cariados, perdidos e obturados segundo o gênero e a faixa etária. FLB, Jequié, 2011.

\begin{tabular}{lcccccc}
\hline \multicolumn{1}{r}{ Variável } & & $\begin{array}{c}\mathrm{n}^{\circ} \\
\text { indivíduos }\end{array}$ & $\begin{array}{c}\text { CPO-D } \\
\text { médio }\end{array}$ & $\begin{array}{c}\text { Cariados } \\
(\%)\end{array}$ & $\begin{array}{c}\text { Perdidos } \\
(\%)\end{array}$ & $\begin{array}{c}\text { Obturados } \\
(\%)\end{array}$ \\
\hline \multirow{2}{*}{ Gênero } & $\mathrm{M}$ & 22 & 28,82 & 7,39 & 81,68 & 0,99 \\
& $\mathrm{~F}$ & 24 & 29,20 & 2,21 & 88,28 & 0,65 \\
Total & & 46 & 29,02 & 4,69 & 85,12 & 0,82 \\
\multirow{2}{*}{ Faixa etária } & $60-74$ & 12 & 26,33 & 4,95 & 75,26 & 2,08 \\
& +75 & 34 & 29,97 & 4,60 & 88,60 & 0,37 \\
Total & & 46 & 29,02 & 4,69 & 85,12 & 0,82 \\
\hline
\end{tabular}

Os valores encontrados para o CPO- indivíduos do gênero masculino apresen-D médio, mediana e desvio padrão fo- taram um baixo percentual de dentes ram, respectivamente, 29,02, 32 e 5,83. obturados em relação aos cariados.

O maior valor encontrado para o CPO-D Ao exame clínico, observou-se um médio foi na faixa etária acima de 75 baixo uso de prótese pelos idosos. Apenas anos. Quando comparados os valores nove (19,56\%) pertenciam a esse grupo, o em relação aos gêneros, observou-se que remete a uma expressiva necessidauma pequena diferença, 29,08\% para o de de próteses, o que pode ser observado masculino e $29,20 \%$ para o feminino. Os na Tabela 2 .

Tabela 2 - Necessidade de prótese de acordo com tipo e arcadas. FLB, Jequié, 2011.

\begin{tabular}{llcc}
\hline & & \multicolumn{2}{c}{ Necessidade } \\
\cline { 3 - 4 } & & $\mathrm{n}$ & $\%$ \\
\hline \multirow{2}{*}{ Total } & Suporior & 35 & 76,09 \\
& Inferior & 32 & 69,56 \\
\multirow{2}{*}{ Parcial removível } & Superior & 03 & 6,52 \\
& Inferior & 12 & 26,08 \\
\hline
\end{tabular}


Todos os idosos necessitavam de algum tipo de prótese. Pôde-se observar uma elevada prevalência de idosos com necessidade de próteses totais, tanto na arcada superior $(76,09 \%)$ quanto na inferior $(69,56 \%)$.

Os altos valores encontrados no CPO-D para dentes perdidos refleti- ram diretamente no Índice Periodontal Comunitário (CPI), que revelou uma percentagem relevante de sextantes excluídos, por apresentarem menos de dois dentes presentes para a realização do exame, especialmente para a faixa etária acima de 75 anos, com $67,41 \%$, como mostra a Tabela 3.

Tabela 3 - Condição periodontal de sextantes em percentual medida pelo Índice Periodontal Comunitário (CPI), segundo a faixa etária. FLB, Jequié, 2011.

\begin{tabular}{lcccccc}
\hline Grupo etário & $\begin{array}{c}\text { Hígido } \\
(\%)\end{array}$ & $\begin{array}{c}\text { Sangramento } \\
(\%)\end{array}$ & $\begin{array}{c}\text { Cálculo } \\
(\%)\end{array}$ & $\begin{array}{c}\text { Bolsa rasa } \\
(\%)\end{array}$ & $\begin{array}{c}\text { Bolsa profunda } \\
(\%)\end{array}$ & $\begin{array}{c}\text { Excluído } \\
(\%)\end{array}$ \\
\hline $60-74$ & 1,81 & 2,17 & 1,45 & 0,00 & 0,00 & 22,82 \\
+75 & 0,72 & 2,54 & 1,08 & 0,00 & 0,00 & 67,41 \\
Total & 2,53 & 4,71 & 2,53 & 0,00 & 0,00 & 90,23 \\
\hline
\end{tabular}

A pior condição mais frequente foi sangramento, observada em $4,71 \%$ dos sextantes avaliados. O mesmo aconteceu com o índice de Perda de Inserção Periodontal (PIP) para sextantes excluídos. A Tabela 4 demonstra que, dentre os sextantes não excluídos, houve a predominância da perda de 0-3 mm, a qual representou $7,61 \%$ dos sextantes avaliados na faixa etária entre 60 e 74 anos. A maior perda observada foi de $9-11 \mathrm{~mm}$, em apenas um indivíduo.

Tabela 4 - Índice de Perda de Inserção Periodontal (PIP) em percentual segundo a faixa etária. FLB, Jequié, 2011.

\begin{tabular}{ccccccc}
\hline $\begin{array}{c}\text { Grupo } \\
\text { etário }\end{array}$ & $\begin{array}{c}\text { Perda } \\
0-3 \mathrm{~mm} \\
(\%)\end{array}$ & $\begin{array}{c}\text { Perda } \\
4-5 \mathrm{~mm} \\
(\%)\end{array}$ & $\begin{array}{c}\text { Perda } \\
6-8 \mathrm{~mm} \\
(\%)\end{array}$ & $\begin{array}{c}\text { Perda } \\
-11 \mathrm{~mm} \\
(\%)\end{array}$ & $\begin{array}{c}\text { Perda } \\
+12 \mathrm{~mm} \\
(\%)\end{array}$ & $\begin{array}{c}\text { Excluído } \\
(\%)\end{array}$ \\
\hline $60-74$ & 4,71 & 0,00 & 0,72 & 0,00 & 0,00 & 22,82 \\
+75 & 2,90 & 1,08 & 0,00 & 0,36 & 0,00 & 67,41 \\
Total & 7,61 & 1,08 & 0,72 & 0,36 & 0,00 & 90,23 \\
\hline
\end{tabular}


Quanto ao tempo de residência dos idosos, a Tabela 5 demonstra que a maioria encontra-se há mais de um ano na instituição. Merece destaque o fato de nove idosos $(20,45 \%)$ residirem há mais de 10 anos na FLB.

Tabela 5 - Tempo de residência dos idosos na FLB, Jequié, 2011.

\begin{tabular}{lcc}
\hline \multicolumn{1}{c}{ Tempo de residência } & $\mathrm{n}^{\circ}$ de indivíduos & $\%$ \\
\hline$<1$ ano & 5 & $11,36 \%$ \\
De 1 a 5 anos & 19 & $41,30 \%$ \\
De 6 a 10 anos & 13 & $28,26 \%$ \\
Acima de 10 anos & 9 & $20,45 \%$ \\
\hline
\end{tabular}

Os idosos analisados foram classificados em independentes e dependentes (incluindo-se aqui os idosos parcialmente dependentes), utilizando como critério suas limitações físicas e psíquicas. $\mathrm{O}$ grau dependente foi o mais frequente, apresentando uma percentagem de 58,70\%, 27 indivíduos (Gráfico 1).

Tabela 6 - Associação entre grau de dependência e uso de prótese, edentulismo dos idosos na FLB, Jequié, 2011.

\begin{tabular}{lcccc}
\hline & & \multicolumn{2}{c}{ Idosos dependentes } & \\
\cline { 3 - 4 } & & Sim & Não & P \\
\hline \multirow{2}{*}{ Uso de prótese } & Sim & 8 & 1 & \multirow{2}{*}{$0,002183^{*}$} \\
& Não & 12 & 25 & \\
Edentulismo & Sim & 12 & 20 & \multirow{2}{*}{$0,216244^{*}$} \\
\hline
\end{tabular}

${ }^{*}$ Teste qui-quadrado de Pearson.

Não foi observada associação estatística entre grau de dependência e edentulismo, nem com necessidade de prótese, mas foi observada associação estatística entre o grau de dependência e o uso de prótese $(\mathrm{p}<0,05)$.

A análise dos prontuários médicos permitiu o estudo das enfermidades dos idosos, das quais se destacaram o diabetes e a hipertensão, com, respectivamente, 9 e 14 indivíduos afetados. Os tipos de enfermidades encontrados podem ser vistos na Tabela 7 , ressaltando-se que 0 mesmo indivíduo pode ser afetado com mais de um tipo de enfermidade. 
Tabela 7 - Acometimento de enfermidades. FLB, Jequié, 2011.

\begin{tabular}{lc}
\hline \multicolumn{1}{c}{ Tipos de enfermidades } & $\begin{array}{c}\mathrm{n}^{\circ} \text { de } \\
\text { enfermidades }\end{array}$ \\
\hline Alcoolismo & 1 \\
Alzheimer & 2 \\
AVE & 1 \\
Câncer & 2 \\
Deficiente visual & 4 \\
Depressão & 1 \\
Diabetes & 9 \\
Hipertensão & 14 \\
Insônia & 1 \\
Problemas neurológicos & 2 \\
Problemas gastrointestinais & 2 \\
Problemas do trato urinário & 2 \\
Nenhuma enfermidade & 3 \\
\hline
\end{tabular}

Não foi observada associação estatística entre a presença de enfermidades e edentulismo.

\section{Discussão}

Os índices epidemiológicos de doenças bucais mais prevalentes, como a cárie, começaram a apresentar melhoras para pessoas mais jovens, devido, principalmente, ao aumento da divulgação em massa de métodos preventivos. No entanto, muitas vezes, não se dá a importância necessária à condição bucal e à necessidade de tratamento da população. (LOPES et al., 2010; SEMAN et al., 2007).

Segundo a OMS, a meta preconizada para o ano 2010 era de que apenas 5\% dos idosos fossem desdentados, o que foge da realidade atual diante dos dados obtidos no presente trabalho e, também, em relação ao que afirmaram Melo et al. (2001), entre outros autores.
Atualmente, observa-se um predomínio de mulheres acima de 60 anos em proporções que aumentam conforme progride a idade. (MASTROENI et al., 2007; RIBEIRO et al., 2011). Entretanto, essa situação não foi encontrada entre os idosos residentes na FLB, onde a proporção entre indivíduos dos dois gêneros não apresentou uma diferença significativa.

O CPO-D médio obtido no presente estudo foi de 29,02, com grande participação do componente "dentes perdidos". Resultado semelhante foi encontrado no estudo de Piuvezam et al. (2011), que relatou CPO-D de 28,08, para idosos institucionalizados de 11 municípios de médio e grande porte, selecionados aleatoriamente nas cinco regiões geográficas do Brasil. Souza et al. (2010), ao avaliarem a influência da saúde bucal no cotidiano de idosos institucionalizados e não institucionalizados da cidade do Recife - PE, obtiveram a média do CPO-D de 30,48 para o primeiro grupo.

Gaião et al. (2009), em seu estudo realizado em Fortaleza - CE, examinou 167 idosos institucionalizados, encontrando resultados semelhantes, com CPO-D médio de 29,7. Reis et al. (2005) também examinaram idosos institucionalizados, em Goiânia, verificando CPO-D médio foi de 30,17. Tais resultados demonstram o alto CPO-D apresentado pela população idosa institucionalizada, uma vez que, conforme os valores encontrados pelo SB Brasil 2010, para a região Nordeste, na faixa etária de 65 a 74 anos, a média do CPO-D foi de 27,10.

A percentagem de $97,28 \%$ para dentes perdidos elevou a média de CPO-D. Em outros estudos presentes na 
literatura (ALCÂNTARA et al., 2011; CARNEIRO et al., 2005; SILVA; VALSECKI JUNIOR, 2000; ROSA et al.; 1992; RIBEIRO et al., 2011), também foi encontrado um alto índice no valor do componente "P", devido à falta de uma prática preventiva por parte dos cirurgiões-dentistas, que, há alguns anos, vêm realizando extrações desnecessárias e eventualmente iatrogênicas, o que fez aumentar o índice de edêntulos no país. Além disso, a Fundação Leu Brito não oferece atendimento odontológico aos idosos.

Em relação aos resultados obtidos no SB 2003, a percentagem para dentes perdidos encontrada na região Nordeste, na faixa etária entre 65 e 74 anos, foi de $92,41 \%$ e a média nacional, de $92,25 \%$.

A perda parcial ou total dos elementos dentais retrata um processo irreversível e cumulativo, refletindo a desigualdade e o sofrimento apresentados pelos idosos da FLB. Essa condição aparece com um dos principais problemas encontrados nessa população, devido às consequências que esse fato pode ter em suas vidas, em relação à saúde. (ALCÂNTARA et al., 2011; SHAMDOL et al., 2008).

A necessidade de prótese total superior e inferior mostrou-se elevada no presente estudo, correspondendo a $76,09 \%$ e $59,56 \%$, respectivamente. Reis et al. (2005) também encontraram altas percentagens para a necessidade de prótese total superior $(71,63 \%)$ e inferior (74,39\%), corroborando os resultados do presente estudo. Shenoy e Hegde (2011) examinaram 133 idosos institucionalizados em Mangalore, na Índia, e observa- ram que 46,6\% dos idosos necessitam de prótese total no arco superior e $41,4 \%$, no arco inferior.

Shrivastav et al. (2011) realizaram um estudo com 177 idosos institucionalizados em Madhya Pradesh, Índia. Ao avaliarem a necessidade do uso de próteses totais, observaram que $16,20 \%$ dos idosos necessitavam no arco superior e $20,50 \%$, no arco inferior.

Resultados parciais do Projeto SB 2010 foram publicados pelo Ministério da Saúde, no qual foram realizadas comparações com os dados obtidos no SB 2003. Dentre os resultados, pôde-se observar que, em relação à necessidade do uso de prótese total, no ano de 2003, 24\% dos idosos a apresentaram em uma arcada, e, no ano de 2010, houve uma queda para $23 \%$. O mesmo aconteceu em relação ao uso de prótese total nas duas arcadas. Em 2003, 16\% apresentaram a necessidade, que, em 2010, caiu para $15 \%$.

Apesar do alto índice de perda dentária, a condição periodontal foi avaliada no presente estudo. Verificou-se um total de $90,23 \%$ de sextantes excluídos, dos idosos examinados, tendo sido o sangramento $(4,71 \%)$ a pior condição mais frequente encontrada no CPI. Quanto ao Índice PIP, os resultados mais frequentes foram para perda de 0-3 $\mathrm{mm}(7,61 \%)$. Tais valores são justificados pelo grande número de idosos desdentados e, até mesmo, grandes perdas dentárias que levaram à exclusão de sextantes.

Quando avaliados CPI e PIP, de acordo com a faixa etária, neste estudo, prevaleceu a condição sangramento para o CPI e, para o PIP, a condição mais frequente, em ambas as faixas 
etárias, foi de 0-3 mm. Carneiro et al. (2005) realizaram um estudo com 293 idosos institucionalizados em São Paulo e observaram que não houve diferença entre as percentagens apresentadas pelo CPI em relação às faixas etárias (65-74 $\mathrm{e} \geq 75$ ), tendo prevalecido a condição cálculo $(94,7 \%)$. Para o PIP, a condição mais frequente foi a perda de $6-8 \mathrm{~mm}$, que também prevaleceu em ambas as faixas etárias.

Corchero e Cepeda (2008), em um estudo realizado em Vigo, Espanha, com 459 idosos institucionalizados em três lares, avaliaram o CPI e encontraram uma elevada percentagem de sextantes excluídos (71\%), tendo sido o sangramento a pior condição mais frequente dos sextantes avaliados, com $13,3 \%$. Os resultados do SB Brasil 2003 demonstraram que a presença de cálculo foi a condição mais frequente no CPI, e, no PIP, prevaleceu sextantes com perda de 0 a $3 \mathrm{~mm}$, assim como no presente estudo.

Em relação ao tempo de residência dos idosos na FLB, neste estudo, foi constatado que a maior parte deles $(40,91 \%)$ encontrava-se na instituição por um período compreendido entre 1 e 5 anos. Contudo, é relevante o fato de nove idosos $(20,45 \%)$ residirem há mais de 10 anos. Resultados aproximados foram encontrados por Gaião et al. (2009), quando verificaram que $53,1 \%$ dos idosos residiam entre 0 a 5 anos na instituição, e 26,9\%, há mais de 10 anos.

Neste estudo, os idosos foram avaliados quanto ao grau de dependência, obtendo-se o seguinte resultado: dependente $(30,43 \%)$, parcialmente dependente $(28,27 \%)$ e independente $(41,30 \%)$.
Colussi et al. (2004) examinaram 277 idosos não institucionalizados no município de Biguaçu - SC, apresentando dados bastante divergentes do presente estudo, obedecendo aos mesmos critérios de avaliação: totalmente dependente $(5,4 \%)$, parcialmente dependente $(26 \%)$ e independente $(68,6 \%)$. Esses resultados mostram que existem perfis diferentes de instituições asilares em todo o país.

Quanto ao acometimento de enfermidades, foram analisados os prontuários médicos disponibilizados pela Fundação Leur Brito. Observou-se que, das doenças crônicas, as mais frequentes foram hipertensão arterial e diabetes, afetando, respectivamente, 14 e 9 idosos. Vale ressaltar que o mesmo indivíduo pode possuir mais de uma enfermidade. Guedes e Silveira (2004), em estudo desenvolvido com 109 idosos de três asilos de Passo Fundo - RS, avaliaram o acometimento de enfermidades de acordo com o grau de dependência (dependentes ou independentes). Os autores observaram que, entre as enfermidades crônicas, as mais frequentes foram as relacionadas a comprometimento mental e hipertensão arterial.

Hamasha et al. (1998), em seu estudo com 175 idosos institucionalizados em Iowa, EUA, avaliaram a associação do acometimento de enfermidades com o edentulismo. Pôde-se observar que os indivíduos desdentados eram mais propensos a ter doenças cardíacas, enquanto os dentados não apresentaram histórico para essas enfermidades. 


\section{Conclusão}

A condição bucal dos idosos residentes na FLB encontra-se comprometida, em decorrência do grande número de dentes perdidos e o baixo uso de prótese. Diante disso, tornam-se necessários o planejamento e a execução de ações em saúde bucal visando a um atendimento integral e equânime desses idosos.

\section{Conditions of oral health of elderly institutionalized}

\section{Abstract}

Given the rapidly aging population and increasing life expectancy, it is clear the need to provide a quality of life with aging. This work aims to verify the oral health status of 46 elderly subjects living in LeurBrito Foundation, the city of Jequié, Bahia. The results showed a $97.28 \%$ of tooth loss, with mean DMFT of 29.02. The need for the use of dentures (76.09\%) and lower (69.56\%) showed poor oral health condition. Periodontal status was shown with the prevalence of bleeding in the $\mathrm{CPI}$, and loss of 0 to $3 \mathrm{~mm}$ in PIP. The degree of dependence, it was observed that $41.30 \%$ of the elderly were independent. At the onset of diseases prevalent hypertension and diabetes. The conditions found in the survey reveals the precarious situation of the oral health of the elderly, emphasizing the importance of actions aimed at health education, and a satisfactory service to provide them a more peaceful age. It is necessary to focus on specific care programs that soon this condition is a positive change.

Keywords: Aging. Health of Institucionalized Elderly. Health Promotion.

\section{Agradecimento}

À direção da Fundação Leur Brito, pela autorização para realização do estudo, e aos integrantes do projeto de extensão Odontoidoso: mão amiga da Universidade Estadual do Sudoeste da Bahia.

\section{Referências}

ALCANTARA, C. M. et al. Estudo comparativo da condição de saúde bucal de idosos não institucionalizados de Governador Valadares - MG, com a meta proposta pela Organização Mundial de Saúde para 2010. Physis Revista de Saúde Coletiva, Rio de Janeiro, v. 21, n. 3, p. 1023-1044, 2011.

BRASIL. Ministério da Saúde. Projeto SB Brasil 2003: condições de saúde bucal da população brasileira 2002-2003 - resultados principais. Brasília: Ministério da Saúde, 2004. Disponível em: <http:// dtr2001.saude.gov.br/editora/produtos/livros/ pdf/05_0053_M.pdf>. Acesso em: 10 mar. 2012 .

Projeto SB 2010: re-

sultados parciais. Disponível em: <http://189.28.128.100/dab/docs/geral/projeto_sb2010_relatorio_final.pdf $>$. Acesso em: 10 mar. 2012.

. Senado Federal. Estatuto do Idoso. Íntegra da Lei n⿳0 10.741 , de 01 de outubro de 2003. Brasília: Centro Gráfico, 2003.

CARNEIRO, R. M. V. et al. Saúde bucal de idosos institucionalizados, zona leste de São Paulo, Brasil, 1999. Caderno de Saúde Pública, Rio de Janeiro, v. 21, n. 6, p. 17091716, 2005.

COLUSSI, C. F.; FREITAS, S. F. T.; CALVO, M. C. M. Perfil epidemiológico da cárie e do uso e necessidade de prótese na população idosa de Biguaçu, Santa Catarina. Revista Brasileira de Epidemiologia, São Paulo, v. 7, n. 1, p. 1313-1320, 2004. 
COLUSSI, C. F.; FREITAS, S. F. T. Aspectos epidemiológicos da saúde bucal do idoso no Brasil. Caderno de Saúde Pública, Rio de Janeiro, v. 18, n. 5, p. 1313-1320, set./out. 2002.

CORCHERO, M. I.; CEPEDA, J. R. G. Oral health in people over 64 years of age, institutionalized in Centres for the Aged in the Vigo Health District Spain, 2005. Medicina Oral, Patología Oral y Cirugía Buca, v. 13, n. 8, p. 523-528, 2008.

GAIÃO, L. R. et al. Poor dental status and oral hygiene practices in institutionalized older people in Northeast Brazil. International Journal of Dentistry, v. 1, n. 6, 2009. Disponível em: <http://www.hindawi.com/ journals/ijd/2009/846081/>. Acesso em: 10 mar. 2012.

GUEDES, J. M.; SILVEIRA, R. C. R. Análise da capacidade funcional da população geriátrica institucionalizada na cidade de Passo Fundo - RS. Revista Brasileira de Ciências do Envelhecimento Humano, Passo Fundo, v. $10, \mathrm{n}$. p. 21, 2004.

HAMASHA, A. A.; HAND, J. S.; LEVY, S. M. Medical conditions associated with missing teeth and edentulism in the institutionalized elderly. Special Care in Dentistry, v. 18, p. 123-127, 1998.

LOPES, M. C.; OLIVEIRA, V. M. B.; FLÓRIO, F. M. Condição bucal, hábitos e necessidade de tratamento em idosos institucionalizados de Araras (SP, Brasil). Ciência \& Saúde Coletiva, Rio de Janeiro, v. 15, n. 6, p. 2949-2954, 2010.

MASTROENI, M. F. et al. Perfil demográfico de idosos da cidade de Joinville, Santa Catarina: estudo de base domiciliar. Revista Brasileira de Epidemiologia, São Paulo, v. 10, n. 2, p. 190-201, 2007.

SHAMDOL, Z. et al. Prevalenceand associated factors of edentulism among elderly muslims in Kota Bharu, Kelantan, Malaysia. Journal of the Islamic Medical Association of North America, v. 40, p. 143-148, 2008.
MELO, N. S. F. O; SETO, E. P. S.; GERMANN, E. R. Medidas de higiene oral empregadas por pacientes da terceira idade. Pesquisa Brasileira de Odontopediatria e Clínica Integrada, João Pessoa, v. 1, n. 3, p. 42-50, 2001.

MONTERO, J.; BRAVO, M.; ALBALADEJO, A. Validation of two complementary oral-health related quality of lifeindicators (OIDP and OSS 0-10 ) in two qualitatively distinct samples of the Spanish population. Health and Quality of Life Outcomes, v. 6, n. 101, p. 1-14, 2008.

OLIVEIRA, D. L.C.; GORETTI, L. C.; PEREIRA, L. S. M. O desempenho de idosos institucionalizados com alterações cognitivas em atividades de vida diária e mobilidade: estudo piloto. Revista Brasileira de Fisioterapia, São Carlos, v. 10, n. 1, p. 91-96, 2006.

OWOTADE, F. J.; OGUNBODEDE, E. O.; LAWAL, A. A. Oral diseases in the elderly, a study in Ile-Ife, Nigeria. Journal of Social Sciences, v. 10, n. 2, p. 105-110, 2005.

PIUVEZAM, G.; LIMA, K. C. Self-perceived oral health status in institutionalized elderly in Brazil. Archives of Gerontology and Geriatrics, v. 5, n. 1, p. 5-11, 2011.

REIS, S. C. G. B. et al. Condição de saúde bucal de idosos institucionalizados em Goiânia - GO, 2003. Revista Brasileira de Epidemiologia, São Paulo, v. 8, n. 1, p. 67-73, 2005.

RIBEIRO, M. T. F. et al. Edentulism and shortened dental arch in Brazilian elderly from the National Survey of Oral Health 2003. Revista de Saúde Pública, São Paulo, v. 45 , n. 6 , p. 817-823, 2011.

RODRIGUES, S. M. et al. Implications of edentulism on quality of life among elderly. International Journal of Environmental Research and Public Health, v. 9, n. 1, p. 100109, 2012.

ROSA, A. G. F. et al. Condições de saúde bucal em pessoas de 60 anos ou mais no município de São Paulo (Brasil). Revista de Saúde Púbica, São Paulo, v. 26, n. 3, p. 155-160, 1992. 
SEMAN, K.; ABDUL MANAF, H.; ISMAIL, A. R. Dental caries experience of elderly people living in "Pondok" in Kelantan. Archives of Orofacial Sciences, v. 2, p. 20-25, 2007.

SHENOY, R. P.; HEGDE, V. Dental prosthetic status and prosthetic need of the institutionalized elderly living in geriatric homes in Mangalore: a pilot study. International Scholarly Research Network, v. 1, n. 3, 2011.

SHRIVASTAV, A. et al. Dental prosthetic status and needs of the residents of geriatric homes in Madhya Pradesh, India. Journal of International Oral Health, v. 3, n. 4, p. 1-4, 2011.

SILVA, S. R. C.; VALSECKI, JUNIOR, A. Avaliação das condições de saúde bucal dos idosos em um município brasileiro. Revista Panamericana de Salud Publica, v. 8, n. 4, p. 268-271, 2000.

SILVA, E. M. M. S.; MARCHIORI, A. V.; FERNANDES, A. U. R. Mudanças fisiológicas e psicológicas na velhice relevantes no tratamento odontológico. Revista Ciência e Extensão, São Paulo, v. 2, n. 1, p. 62, 2005.

SILVA, S. O. Saúde bucal do idoso institucionalizado em dois asilos de Passo Fundo - RS. Revista Gaúcha de Odontologia, Porto Alegre, v. 56, n. 3, p. 303-308, 2008.

SOUZA, E. H. A. Impacto da saúde bucal no cotidiano de idosos institucionalizados e não institucionalizados da cidade do Recife (PE, Brasil). Ciência \& Saúde Coletiva, Rio de Janeiro, v. 15, n. 6, p. 2955-2964, 2010.

SOUZA, V. M. S.; PAGANI, C.; JORGE, A. L. C. Odontogeriatria: sugestão de um programa de prevenção. Revista da Faculdade de Odontologia de São José dos Campos, São José dos Campos, v. 4, n. 1, p. 56-62, 2001. 